\title{
Hidden Fermionic Symmetry in Conformal Topological Field Theories
}

\author{
Tohru Eguchi', Shinobu Hosono ${ }^{1}$ and Sung-Kil Yang ${ }^{2}$ \\ ${ }^{1}$ Institute for Theoretical Physics, University of California, Santa Barbara, CA 93106, USA \\ and Department of Physics, University of Tokyo, Tokyo, Japan 113 \\ ${ }^{2}$ National Laboratory for High Energy Physics (KEK), Ibaraki, Japan 305
}

Received January 30, 1991

\begin{abstract}
We point out that bosonic conformal coset models $G_{l} \times G_{k} / G_{l+k}$ for all semi-simple Lie algebras $G$ have a hidden fermionic symmetry at $l=0$ (the central charge $=0$ ) and may be interpreted as twisted versions of some superconformal theories.
\end{abstract}

In a previous communication we pointed out that a series of $c=0$ conformal models of the $S U(2)$ GKO coset construction may be interpreted as a twisted version of $N=2$ minimal superconformal theories [1]. When a conformal model has a vanishing central charge $c=0$, it no longer depends on the complex structure of the Riemann surface and becomes a topological field theory. It turned out [2] that the twisted $N=2$ minimal theories reproduce the results of the matrix models [3] when coupled to gravity and thus twisted $N=2$ models appear to play some basic role in the theory of 2 dimensional gravity.

In this article we would like to generalize our previous treatment and ask if a bosonic coset model $G_{l} \times G_{k} / G_{l+k}$ based on a general Lie algebra $G$ has a hidden fermionic symmetry at $c=0(l=0)$. We point out that in the case of a Lie algebra $G$ which yields a hermitian symmetric space $G / H \times U(1)$ when a suitable subgroup $H$ is chosen, $G$-coset model is identified as the twisted version of superconformal (Kazama-Suzuki) model [4] based on the hermitian symmetric space $G / H \times U(1)$. On the other hand in the case of Lie algebras $G_{2}, F_{4}, E_{8}$ which do not yield hermitian symmetric spaces, we find a new way of constructing fermionic symmetry. We identify the bosonic coset models as the twisted version of the $N=1$ supercoset theories based on the Wolf spaces $G / H \times S U(2)$. Thus all bosonic coset models with vanishing central charge have a hidden fermionic symmetry. Furthermore some of the coset models are identified as the twisted versions of not one but several superconformal theories at the same time.

Let us first briefly recall our previous observation on the $S U(2) \mathrm{GKO}$ coset 
theories,

$$
S U(2)_{l} \times S U(2)_{k} / S U(2)_{l+k},
$$

where $S U(2)_{l}$ denote the affine $S U(2)$ algebra at level-l. In the Coulomb gas representation the stress-tensor of the theory is given by

$$
T(z)=T_{Z_{k}}(z)-\frac{1}{2}(\partial \phi(z))^{2}+i \alpha_{0} \partial^{2} \phi(z),
$$

where $\alpha_{0}=\left(p^{\prime}-p\right) / \sqrt{2 k p p^{\prime}}, p=l+2$ and $p^{\prime}=l+k+2 . T_{Z_{k}}(z)$ is the stress-tensor of the level- $k S U(2)$-parafermion theory and $\phi$ is the free bosonic field. In terms of the parafermion fields $\psi_{1}(z), \psi_{1}^{*}(z)$ with dimensions $1-1 / k$, the screening operators of the theory are expressed as

$$
S_{+}(z)=\psi_{1}(z) e^{i \alpha_{+} \phi(z)}, \quad S_{-}(z)=\psi_{1}^{*}(z) e^{i \alpha_{-} \phi(z)} .
$$

where $\alpha_{+}=\sqrt{2 p^{\prime} / k p}, \alpha_{-}=-\sqrt{2 p / k p^{\prime}}$. Screening operators (3) have dimension 1 and have the nilpotency property and are interpreted as BRST operators. They play a basic role in reducing the bosonic Fock space to the irreducible representation space of the Virasoro algebra which are given by BRST cohomology classes [5].

Consider now the $l=0$ case. We obtain a series of $c=0$ theories labeled by $k=1,2,3, \ldots$, and we expect that the stress-tensor is written as a BRST commutator

$$
T(w)=\left\{\int Q(z) d z, Q^{*}(w)\right\} .
$$

Here the BRST current $Q$ is a dimension- 1 operator and the BRST partner of the stress-tensor, $Q^{*}$, has a dimension $h=2$. It is easy to check that the $(3,1)$ operator

$$
\Phi_{3,1}(z)=\psi_{1}^{*}(z) e^{-i \alpha+\phi(z)}
$$

has a dimension $h=2$ exactly at $l=0\left(\alpha_{0}=\frac{1}{2} \sqrt{\frac{k}{(k+2)}}, \alpha_{+}=\sqrt{(k+2) / k}\right)$ and its anti-commutator with the screening operator $S_{+}$reproduces the energymomentum tensor. Thus we identify

$$
S_{+}=Q, \Phi_{3,1}=Q^{*} .
$$

On the other hand it is well-known that the stress-tensor and the $U(1)$ current of the level- $k$ minimal $N=2$ superconformal models has the form [6],

$$
\begin{aligned}
T_{N=2}(z) & =T_{Z_{k}}(z)-\frac{1}{2}(\partial \phi(z))^{2}, \\
J(z) & =2 i \alpha_{0} \partial \phi(z),
\end{aligned}
$$

where $\alpha_{0}=\frac{1}{2} \sqrt{k /(k+2)}$. Thus we find the basic relation

$$
T_{c=0}(z)=T_{N=2}(z)+\frac{1}{2} \partial J(z) .
$$

Operators $Q, Q^{*}$ have dimension $3 / 2$ and $U(1)$ charge \pm 1 with respect to (7), (8) and are identified as the $N=2$ supercurrents. Equation (4) is then nothing but the defining relation of the $N=2$ algebra.

Thus the $c=0 S U(2)$ coset models are interpreted as $N=2$ minimal models with their stress-tensor twisted by the $U(1)$ current. BRST invariant observables 
of the $N=2$ twisted models are given by the chiral ring [7] of $N=2$ (untwisted) theories.

Now we would like to extend the previous analysis to arbitrary coset models $G_{l} \times G_{k} / G_{l+k}$. The Coulomb gas representation for the coset model consists of the $G$-parafermion theory of level $k[8]$ and the free bosons $\phi \equiv\left(\phi_{1}, \phi_{2}, \ldots, \phi_{r}\right)$ with $r=\operatorname{rank} G$. The energy-momentum tensor reads

$$
T_{G}(z)=T_{Z_{k}(G)}(z)-\frac{1}{2}(\partial \phi(z))^{2}+i \alpha_{0} \rho_{G} \partial^{2} \phi(z),
$$

where $\rho_{G}$ is half the sum of positive roots of $G, \alpha_{0}=\left(p^{\prime}-p\right) / \sqrt{k p p^{\prime}}, p=g+l$ and $p^{\prime}=g+k+l$. Here $g$ is the dual Coxeter number of $G$. The Virasoro central charge of $(10)$ is

$$
c=\frac{k \operatorname{dim} G}{k+g}-12 \alpha_{0}^{2} \rho_{G}^{2} .
$$

A coset pair $(l, k)=(l, 1)$ describes the usual $W_{G}$ algebra [9]. For the simply laced algebras the $k=1$ parafermion theory is trivial, however, it is non-trivial for the non-simply laced case. For $G=B_{n}$ the level-1 parafermion becomes a free fermion introduced in [10]. In the $G$-parafermion theory the parafermion field $\psi_{\alpha}(z)$ is defined for each root $\alpha$ among which those associated with the simple roots $\alpha_{i}(i=1,2, \ldots, r)$ play the basic role. The screening operators are written as

$$
S_{\alpha_{l}}(z)=\psi_{\alpha_{i}}(z) e^{i \alpha+\alpha_{\imath} \phi(z)} \quad(i=1,2, \ldots, r),
$$

where the dimension of $\psi_{\alpha_{i}}$ is $1-\alpha_{i}^{2} / 2 k$ and $\alpha_{+}=\sqrt{\frac{p^{\prime}}{k p}}, \alpha_{-}=-\sqrt{\frac{p}{k p^{\prime}}}$ (there exists another class of screening operators $\psi_{-\alpha_{l}}(z) e^{i \alpha_{-} \phi(z)}$ which feature in the offcritical perturbation theory of coset models [11]).

At $c=0$

$$
\alpha_{0}=\sqrt{\frac{k}{g(g+k)}}, \quad \alpha_{+}=\sqrt{\frac{g+k}{g k}}, \quad \alpha_{-}=-\sqrt{\frac{g}{k(g+k)}},
$$

and we have a $G$-series of $c=0$ theories labeled by $k=1,2, \cdots$. In order to express the stress-tensor as a BRST commutator we look for an operator which acquires the dimension $h=2$ right at the "topological" point (13). It turns out that a vertex operator with exponent $-\theta, \theta$ being the highest root of $G$, has exactly this property. Namely the operator,

$$
\psi_{-\theta} e^{-i \alpha_{+} \theta \phi}
$$

has a dimension $h=2$ at $c=0$. Equation (14) is thus the candidate for the operator $Q^{*}$ and (12) are the candidates for $Q$. We use the convention $\theta^{2}=2$ in this paper.

Let us now consider the first case when the Lie algebra $G$ yields a hermitian symmetric space (HSS) $G / H \times U(1)$ when it is quotiented by its suitable subgroup $H \times U(1)$. This is the case with $G=A_{n}, B_{n}, C_{n}, D_{n}(n \geqq 1), E_{6}, E_{7}$. See Fig. 1 . Some simple examples have already been discussed by Lerche [12]. In these cases, we choose any simple root $\alpha_{j}$ out of $\alpha_{1}, \cdots, \alpha_{r}$ which carries the Kac-label 1 in the Dynkin graph of $G$ (Kac-labels $n_{i}$ are defined as the expansion coefficients of the 

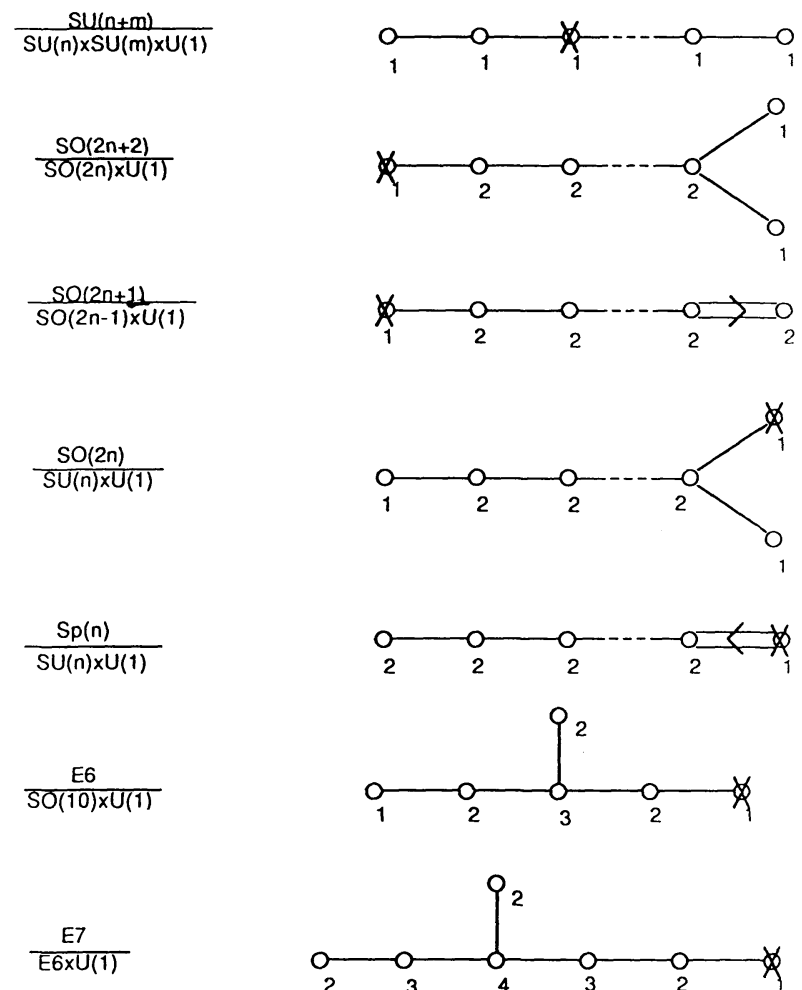

Fig. 1. Hermitian symmetric spaces.

highest root into the sum of simple roots $\theta=\sum_{i=1}^{r} n_{i} \alpha_{i}$. Thus the condition on $\alpha_{j}$ is $\left.n_{j}=1\right)$. We then identify

$$
Q(z)=\psi_{\alpha_{J}}(z) e^{i \alpha+\alpha_{J} \phi(z)}, \quad Q^{*}(z)=\psi_{-\theta}(z) e^{-i \alpha+\theta \phi(z)} .
$$

One may check that in fact

$$
T_{G}(w)=\left\{\int \psi_{\alpha_{j}}(z) e^{i \alpha_{-} \alpha_{j} \phi(z)} d z, \psi_{-\theta}(w) e^{-i \alpha_{+} \theta \phi(w)}\right\},
$$

where $T_{G}(w)$ is given by (10). Calculation of the anti-commutator (16) will be described later. The bosonic form of supercharge operators (15) was first suggested in [13]. The Dynkin graph of the subgroup $H$ is obtained by deleting the vertex $\alpha_{j}$ from the Dynkin graph of $G$. The $U(1)$ direction perpendicular to $H$ is given by the vector $\rho_{G}-\rho_{H}\left(\rho_{H}\right.$ is half the sum of positive roots of $\left.H\right)$. If one defines the $U(1)$ current as [13].

$$
J(z)=2 \mathrm{i} \alpha_{0}\left(\rho_{G}-\rho_{H}\right) \partial \phi(z),
$$

$Q, Q^{*}$ has charge \pm 1 while the screening operators (12) with $i \neq j$ have a vanishing $U(1)$ charge. Now $T_{G}(z)$ is decomposed as

$$
\begin{aligned}
T_{G}(z) & =T_{N=2}+\frac{1}{2} \partial J(z), \\
T_{N=2}(z) & =T_{Z_{k}}(z)-\frac{1}{2}(\partial \phi(z))^{2}+i \alpha_{0} \rho_{H} \partial^{2} \phi(z),
\end{aligned}
$$


and we can identify the stress-tensor $T_{N=2}$ of $N=2$ theory. The central charge of $T_{N=2}$ is given by

$$
c_{N=2}=\frac{k \operatorname{dim} G}{k+g}-12 \alpha_{0}^{2} \rho_{H}^{2} .
$$

Using the Freudental-de Vries formula and a known relation for HSS, $h \operatorname{dim} H=3 g / 2(\operatorname{dim} H+1)-\frac{1}{2} g \operatorname{dim} G$ ( $h$ is the dual Coxeter number of $\left.H\right),(20)$ can be rewritten as

$$
\begin{aligned}
c_{N=2}= & \frac{k \operatorname{dim} G}{k+g}+\frac{1}{2}(\operatorname{dim} G-\operatorname{dim} H-1) \\
& -\frac{(k+g-h) \times \operatorname{dim} H}{k+g}-1 .
\end{aligned}
$$

We note that (21) is the formula for the central charge of Kazama-Suzuki (KS) models [4] based on the HSS $G / H \times U(1)$. Thus $T_{N=2}$ is the bosonized version of the stress-tensor of KS model. Identification of $T_{N=2}(19)$ as the stresstensor of KS model can be made directly in the case $k=1$. Let us consider, for instance, the case of Grassmannian $S U(n+m) / S U(n) \times S U(m) \times U(1)$. We first obtain using identities of conformal embedding [14]

$$
\begin{aligned}
& \text { Super }\left(\frac{S U(n+m)}{S U(n) \times S U(m) \times U(1)}\right)_{k=1} \\
& =\frac{S U(n+m)_{1} \times F(n m)}{S U(n)_{m+1} \times S U(m)_{n+1} \times U(1)} \\
& \approx \frac{S U(n)_{1} \times S U(m)_{1} \times U(1) \times S U(n)_{m} \times S U(m)_{n} \times U(1)}{S U(n)_{m+1} \times S U(m)_{n+1} \times U(1)} \\
& \approx W_{n}(m) \times W_{m}(n) \times U(1),
\end{aligned}
$$

where $F(n m)$ denotes the algebra of $n \times m$ free complex fermions and $W_{n}(m)$ is the $S U(n)-W$ algebra at level $m, W_{n}(m)=\frac{S U(n)_{1} \times S U(n)_{m}}{S U(n)_{m+1}}$. We then use the standard bosonized form for the stress-tensor of the $W$-algebra [9],

$$
\begin{gathered}
T_{W_{n}}=-\frac{1}{2}(\partial \phi)^{2}+i \alpha_{0} \rho_{S U(n)} \partial^{2} \phi, \\
T_{W_{m}}=-\frac{1}{2}(\partial \phi)^{2}+i \alpha_{0} \rho_{S U(m)} \partial^{2} \phi .
\end{gathered}
$$

Scalar fields $\phi$ have $n-1$ and $m-1$ components in (23), (24), respectively. Equations (23) and (24) together with $T_{U(1)}=-\frac{1}{2}(\partial \Phi)^{2}\left(\Phi=\sqrt{\frac{c_{N=2}}{3}} \frac{2 \alpha_{+}}{n m}\left(\rho_{G}-\rho_{H}\right) \phi\right)$
give

$$
T_{N=2}=T_{W_{n}}+T_{W_{m}}+T_{U(1)} .
$$

Note the operators $Q, Q^{*}$ factor into products of primary fields of the algebras $T_{W_{n}}, T_{W_{m}}, T_{U(1)}$,

$$
\begin{aligned}
e^{i \alpha_{+} \alpha_{n} \phi} & =e^{i \alpha_{+}\left(-\Lambda_{n-1}^{n}-\Lambda_{1}^{m}+2 / n m\left(\rho_{G}-\rho_{H}\right)\right) \phi}, \\
e^{-i \alpha_{+} \theta \phi} & =e^{i \alpha_{+}\left(-\Lambda_{1}^{n}-\Lambda_{n-1}^{m}-2 / n m\left(\rho_{G}-\rho_{H}\right)\right) \phi},
\end{aligned}
$$


where $\Lambda_{1}^{n}, \Lambda_{n-1}^{n}$ are the fundamental weights for the vector and $(n-1)$-fold antisymmetric tensor representation of $S U(n)$, respectively. We denote

$$
\begin{aligned}
& \psi_{S U(n)} \equiv \exp \left(-i \alpha_{+} \Lambda_{1}^{n} \phi\right), \\
& \psi_{S U(n)}^{*} \equiv \exp \left(-i \alpha_{+} \Lambda_{n-1}^{n} \phi\right), \\
& \psi_{U(1)} \equiv \exp \left(i \alpha_{+} \frac{2}{n m}\left(\rho_{G}-\rho_{H}\right) \phi\right) .
\end{aligned}
$$

Then $Q=\psi_{S U(n)}^{*} \psi_{S U(m)} \psi_{U(1)}$ and $Q^{*}=\psi_{S U(n)} \psi_{S U(m)}^{*} \psi_{U(1)}^{*}$ are conjugate to each other.

The operator product expansion (OPE) of $Q, Q^{*}$ can be calculated using the OPE of its component fields

$$
\begin{aligned}
\psi_{S U(n)}(z) \psi_{S U(n)}^{*}(w) & \sim \frac{\text { const. }}{(z-w)^{2 h_{n}}}\left\{1+\frac{2 h_{n}}{c_{n}} T_{W_{n}}(w)(z-w)^{2}+\ldots\right\} \\
\psi_{S U(m)}(z) \psi_{S U(m)}^{*}(w) \sim & \frac{\text { const. }}{(z-w)^{2 h_{m}}}\left\{1+\frac{2 h_{m}}{c_{m}} T_{W_{m}}(w)(z-w)^{2}+\ldots\right\} \\
\psi_{U(1)}(z) \psi_{U(1)}^{*}(w) \sim & \frac{\text { const }}{(z-w)^{2 h_{U(1)}}}\left\{1+\frac{3}{c_{N=2}} J(z)(z-w)\right. \\
& \left.+\frac{2 h_{U(1)}}{c_{U(1)}}\left(T_{U(1)}(z)+\frac{3 c_{U(1)}}{2 h_{U(1)} c_{N=2}} \frac{1}{2} \partial J(z)\right)(z-w)^{2}+\ldots\right\}
\end{aligned}
$$

where $h_{n}\left(h_{m}\right)$ is the dimension of $\psi_{S U(n)}\left(\psi_{S U(m)}\right)$ referring to the stress-tensor $T_{W_{n}}$ $\left(T_{W_{m}}\right) \cdot c_{n}\left(c_{m}\right)$ is the central charge of $T_{W_{n}}\left(T_{W_{m}}\right)$. Similarly $h_{U(1)}$ is the dimension of the $U(1)$ vertex operator $\psi_{U(1)}$ and the $U(1)$-current $J$ is defined by

$$
J=i \frac{c_{N=2}}{3} \frac{2 \alpha_{+}}{n m}\left(\rho_{G}-\rho_{H}\right) \partial \phi .
$$

Note that since the $W_{n}, W_{m}$ algebras do not contain $U(1)$ current, the OPE (31), (32) do not have a term at the first order in $(z-w)$-expansion. The coefficient $2 \mathrm{~h} / \mathrm{c}$ in front of the $2^{\text {nd }}$ order term is fixed by the associativity of the operator algebra [6].

After a simple computation we find

$$
\begin{aligned}
h_{n} & =\frac{1}{2} \frac{g+1}{g}\left(1-\frac{1}{n}\right)+\frac{1}{2 g}(n-1), \\
h_{m} & =\frac{1}{2} \frac{g+1}{g}\left(1-\frac{1}{m}\right)+\frac{1}{2 g}(m-1), \\
h_{U(1)} & =\frac{g+1}{2 n m},
\end{aligned}
$$

and

$$
c_{n}=(n-1)\left(1-\frac{n(n+1)}{g(g+1)}\right), \quad c_{m}=(m-1)\left(1-\frac{m(m+1)}{g(g+1)}\right)
$$


where $g=n+m$. Thus

$$
\frac{h_{n}}{c_{n}}=\frac{h_{m}}{c_{m}}=\frac{h_{U(1)}}{c_{U(1)}}=\frac{\frac{3}{2}}{c_{N=2}} .
$$

(Note that $h_{n}+h_{m}+h_{U(1)}=3 / 2, c_{n}+c_{m}+c_{U(1)}=c_{N=2}$.) Hence

$$
Q(z) Q^{*}(w) \sim \frac{\text { const. }}{(z-w)^{3}}\left\{\frac{2}{3} c_{N=2}+2 J(z)(z-w)+2\left(T(z)+\frac{1}{2} \partial J(z)\right)(z-w)^{2}+\cdots\right\},
$$

and we recover the $N=2$ algebra.

It is somewhat surprizing that the anti-commutator (16) gives the same result independent of $\alpha_{j}$ as far as it is chosen from the vertices with Kac-label 1. This can, however, be understood as due to the diagram automorphism which permutes vertices with Kac-label 1. The stress-tensor should be invariant under such transformations. Equation (16) implies that a bosonic coset model with $c=0$ "ramificates" in general under untwisting operation and generates several inequivalent $N=2$ theories.

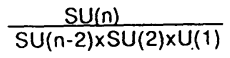

$\frac{S O(n)}{S O(n-4) \times S U(2) \times S U(2)}$

$\frac{S p(n)}{S p(n-1) \times S U(2)}$
$\frac{G 2}{S U(2) \times S U(2)}$
$\frac{F 4}{S p(3) \times S U(2)}$
$\frac{E 6}{S U(6) \times S U(2)}$
$\frac{E 7}{S O(12) \times S U(2)}$

$\frac{E 8}{E 7 \times S U(2)}$
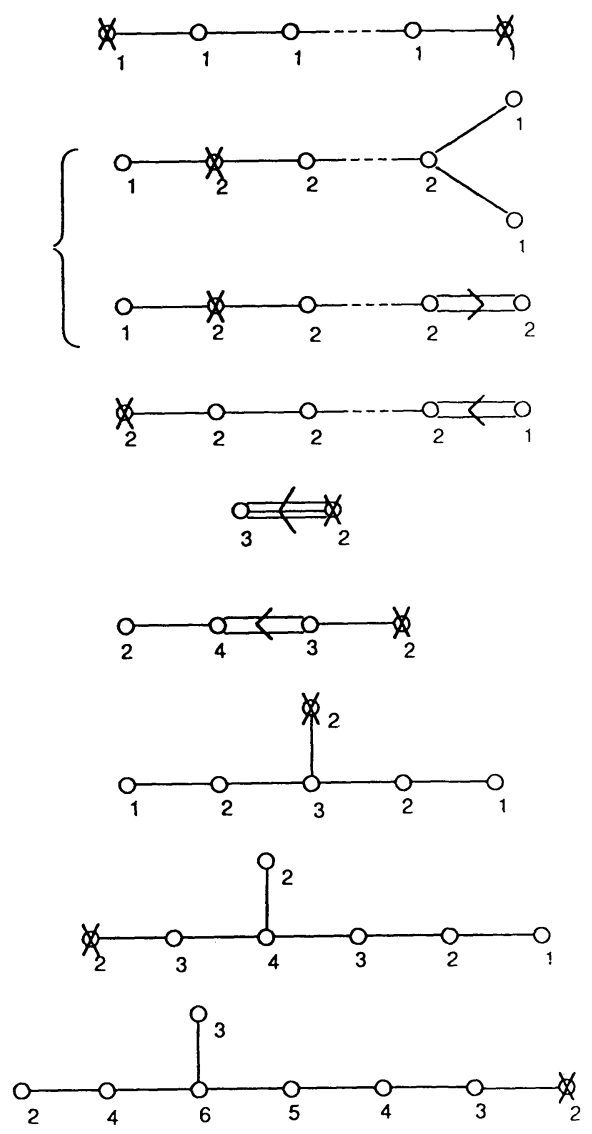

Fig. 2. Wolf spaces 
Now let us turn to the Lie algebras $G_{2}, F_{4}, E_{8}$ which do not have vertices with label 1 in their Dynkin diagrams (see Fig. 2). In these cases we can consider the following construction; first we take the highest root $\theta$ and define as $S U(2)$ subgroup by $E_{\theta}, E_{-\theta},\left[E_{\theta}, E_{-\theta}\right]$, where $E_{\theta}\left(E_{-\theta}\right)$ is the lowering (raising) operator. We then define a subgroup $H$ whose root vectors span an orthogonal subspace to $\theta$ in the root space of the group $G$. Then $G / H \times S U(2)$ gives a Wolf space, a symmetric space with a quaternionic structure [15]. In the case of a Wolf space the Weyl vector $\rho_{G}$ has the following decomposition:

$$
\rho_{G}=\rho_{H}+(1-g) \rho_{S U(2)}, \quad \rho_{S U(2)}=-\theta / 2 .
$$

Then the $c=0$ stress-tensor decomposes as

$$
T_{c=0}=T_{Z_{k}(G)}-\frac{1}{2}(\partial \phi)^{2}+i \alpha_{0}\left(\rho_{H}+\rho_{S U(2)}\right) \partial^{2} \phi+i \alpha_{0}(-g) \rho_{S U(2)} \partial^{2} \phi .
$$

The sum of the first three terms in (42) is identified as the stress-tensor of the supercoset model based on the Wolf-space $G / H \times S U(2)$ which has $N=1$ superconformal symmetry,

$$
T_{N=1}=T_{Z_{k}(G)}-\frac{1}{2}(\partial \phi)^{2}+i \alpha_{0}\left(\rho_{H}+\rho_{S U(2)}\right) \partial^{2} \phi .
$$

Its central charge is given by

$$
c=\frac{6 k(g-2)}{k+g} .
$$

The above construction (43) is not limited to the cases of $G_{2}, F_{4}$ and $E_{8}$ and in fact works for any Lie algebra. Thus the bosonic coset models with $c=0$ have at least $N=1$ hidden fermionic symmetry.

The $N=1$ supercharge operator is given by

$$
Q(z)=\psi_{\gamma} e^{i \alpha+\gamma \phi(z)},
$$

where $\gamma$ is the unique simple root of $G$ which has a non-zero inner product with $\theta$. $\gamma$ has a Kac-label 2 as we see in Fig. 2. (This is except for the case of $G=S U(n)$. In the $S U(n)$ case there are two simple roots $\gamma_{1}, \gamma_{2}$ with non-zero inner product with $\theta$ and both have label 1. Supercharge operator is defined by $\left.Q=\psi_{\gamma_{1}} e^{i \alpha+\gamma_{1} \phi}+\psi_{\gamma_{2}} e^{i \alpha+\gamma_{2} \phi}\right)$. $Q$ has a dimension $h=3 / 2$ referring to $T_{N=1}(43)$. We note that, unlike the HSS case, where $e^{-i \alpha+\theta \phi}$ was conjugate to $e^{i \alpha+\gamma \phi}, e^{-i \alpha+\theta \phi}$ is now an $S U(2)$ current operator and has $h=1$ when referred to $T_{N=1}$.

OPE of $Q$ with itself can be evaluated as before by factoring $Q$ into a product of fields and computing OPE for each of its components. Let us consider the case of the A-D-E algebra at $k=1$ for simplicity. In this case the para-fermion fields are absent and the stress-tensor is decomposed as

$$
T_{N=1}=T_{W(H)}+T_{W(S U(2))} .
$$

Corresponding to the decomposition

$$
\gamma=\theta / 2-w
$$

where $w$ is the highest weight of some fundamental representation of $H, Q$ is factored as

$$
Q=\psi_{S U(2)} \psi_{H},
$$




$$
\psi_{S U(2)}=\exp \left(-i \frac{\alpha_{+}}{\sqrt{2} \Phi}\right), \quad \psi_{H}=\exp \left(-i \alpha_{+} w \phi\right) .
$$

Here $\Phi=\sqrt{2} \rho_{S U(2)} \phi . \psi_{S U(2)}$ is identified as the $(2,1)$ operator of the Virasoro algebra $T_{W(S U(2))}=-\frac{1}{2}(\partial \Phi)^{2}+i 1 / \sqrt{2} \alpha_{0} \partial^{2} \Phi$ with the central charge $c=1-6 / g(g+1)$. In the case of $\mathrm{D}$ and $\mathrm{E}$ algebras, $w$ is a weight of some self-conjugate representation and $\psi_{S U(2)}, \psi_{H}$ are both self-conjugate fields. In the case of A-type algebras, on the other hand, $w_{1}=\theta / 2-\gamma_{1}$ and $w_{2}=\theta / 2-\gamma_{2}$ are weights of conjugate representations. Thus fields $\psi_{H, i}$ defined by $e^{-i \alpha+w_{i} \phi}$ are conjugate to each other. Hence the $N=1$ supercharge operator $Q=\psi_{S U(2)}\left(\psi_{H, 1}+\psi_{H, 2}\right)$ is a self-conjugate field. Using the OPE's as in (31), (32) and

$$
\frac{h_{S U(2)}}{c_{S U(2)}}=\frac{h_{H}}{c_{H}}=\frac{g+1}{4(g-2)}=\frac{3 / 2}{c_{N=1}},
$$

we find

$$
Q(z) Q(w) \sim \frac{\text { const. }}{(z-w)^{3}}\left(\frac{2}{3} c_{N=1}+2 T_{N=1}(w)(z-w)^{2}+\cdots\right),
$$

which is the $N=1$ algebra.

In the above we have evaluated the crucial commutation relations (40), (51) using the OPE of the theory of $W$-algebra. If instead we try to compute them directly using the free-field realization of supercharge operators, we must take into account the screening operators of the $c=0$ theory. The relevant BRST operator $Q_{\mathrm{BRST}}$, for instance, is given by a multiple-integral of the screening operators

$$
Q_{\mathrm{BRST}}=\prod_{j=1}^{r} \int \cdots \int \psi_{\alpha_{j}}\left(z_{j, 1}\right) e^{i \alpha+\alpha_{j} \phi\left(z_{j, 1}\right)} \cdots \psi_{\alpha_{j}}\left(z_{j, n_{j}}\right) e^{i \alpha_{+} \alpha_{j} \phi\left(z_{j}, n_{j}\right)} d z_{j, 1} \cdots d z_{j, n_{j}}
$$

where $n_{j}$ is the Kac-label of the vertex $\alpha_{j}$ and the product over $j$ runs over all the simple roots of $G$. The integration contour of (52) is chosen as in [5]. The stresstensor of the $c=0$ theory will then be expressed as

$$
T_{c=0}(w)=\left\{Q_{\mathrm{BRST}}, \psi_{-\theta}(w) e^{-i \alpha_{+} \theta \phi(w)}\right\} .
$$

Let us consider the relation (53) in the case of $\mathrm{HSS}=G / H \times U(1)$, where the subgroup $H$ is obtained from $G$ by deleting a simple root $\gamma=\alpha_{j}$. In this case (53) is reduced to our previous formula (16),

$$
T_{c=0}(w)=\left\{\oint \psi_{\gamma}(z) e^{i \alpha+\gamma \phi(z)} d z, \psi_{-\theta}(w) e^{-i \alpha+\theta \phi(w)}\right\} .
$$

This is because $n_{\gamma}=1$ in this case and we have used the fact that the operators $\psi_{\alpha_{i}} e^{i \alpha+\alpha_{i} \phi}(i \neq j)$ are also the screening operators of the $N=2$ theory and thus may be identified as identity operators. On the other hand, if we consider the case of an $N=1$ theory based on the Wolf space $G / H \times S U(2)$, where $H$ is obtained from $G$ by deleting a vertex $\gamma$, we obtain

$$
T_{c=0}=\left\{\iint \psi_{\gamma}\left(z_{1}\right) e^{i \alpha+\gamma \phi\left(z_{1}\right)} \psi_{\gamma}(z) e^{i \alpha+\gamma \phi\left(z_{2}\right)} d z_{1} d z_{2}, \psi_{-\theta}(w) e^{-i \alpha+\theta \phi(w)}\right\} .
$$

This is because $n_{\gamma}=2$ in this case and the fields $\psi_{\alpha_{i}} e^{i \alpha+\alpha_{1}}\left(\gamma \neq \alpha_{i}\right)$ are the screening 
operators of the $N=1$ theory. The formula (53) was first evaluated by Mizoguchi in the case $G=S U(3)$ and has been checked in some other examples.

Acknowledgements. T.E. and S.H. would like to thank Institute for Theoretical Physics, University of California, Santa Barbara for its hospitality. S. K. Yang thanks Niels Bohr Institute where a part of this work was done. This research was supported in part by the National Science Foundation under grant No. PHY-04035, supplemented by funds from the National Aeronautics and Space Administration.

\section{References}

1. Eguchi, T. Yang, S. -K.: Mod. Phys. Lett. A4, 1653 (1990)

2. K. Li, Caltech-preprint: CALT-68-1662, 1990; Dijkgraaf, R., Verlinde, H., Verlinde, E.: Princeton preprint PUPT-1204, IASSNS-HEP-90/71, 1990

3. Brezin, E., Kazakov, V.: Phys. Lett. B236, 144 (1990); Douglas, M., Shenker, S.: Nucl. Phys. B335, 635 (1990); Gross, D. J. Migdal, A.: Phys. Rev. Lett. 64, 127 (1990); Douglas, M.: Phys. Lett. B238, 207 (1990); Witten, E.: Nucl. Phys. B340, 281 (1990); Distler, J.: Nucl. Phys. B342, 523 (1990); Dijkgraaf, R., Witten, E.: Nucl. Phys. B342, 486 (1990); Verlinde E., Verlinde, H.: Nucl. Phys. B348, 457 (1990); Dijkgraaf, R., Verlinde, H., Verlinde, E.: Nucl. Phys. 348, 435 (1991); Fukuma, M., Kawai, H., Nakayama, R.: Tokyo preprint UT-562, 1990

4. Kazama, Y., Suzuki, H.: Nucl. Phys. B321, 232 (1989); Phys. Lett. B216, 112 (1989)

5. Felder, G.: Nucl. Phys. B317, 215 (1989); Felder, G., Fröhlich, J., Keller, G.: Commun. Math. Phys. 124, 647 (1989); Bouwknegt, P., McCarthy, J., Pilch, K.: Commun. Math. Phys. 131, 125 (1990)

6. Zamolodchikov, A. B., Fateev, V. A.: Zh. Eksp. Teor. Fiz. 89, 380 (1985); [Sov. Phys. JETP 62, 215 (1985)]; Zh. Eksp. Teor. Fiz. 90, 1553 (1986); [Sov. Phys. JETP 63, 913 (1986)]; Qiu, Z.: Phys. Lett. B188, 207 (1987)

7. Lerche, W., Vafa, C., Warner, N. P.: Nucl. Phys. B324, 427 (1989); Hosono, S., Tsuchiya, A.: Tokyo preprint UT-561 May (1990); Gepner, D.: Weizmann Institute preprint WIS90/47/Ph (1990)

8. Gepner, D.: Nucl. Phys. 290, 10 (1987); Ninomiya, M., Yamagishi, K.: Phys. Lett. B183, 323 (1987)

9. Fateev, V. A., Zamolodchikov, A. B.: Nucl. Phys. B280, 644 (1987); Fateev, V. A., Lykyanov, S. L.: Int. J. Mod. Phys. A3, 507 (1988)

10. Lykyanov, S. L., Fateev, V. A.: Kiev preprints ITP-88-74p, 75p and 76p (1988)

11. Eguchi, T., Yang, S. -K.: Phys. Lett. B224, 373 (1989); Phys. Lett. B235, 282 (1989) Hollowood, T., Mansfield, P.: Phys. Lett. B226, 73 (1989)

12. Lerche, W.: Phys. Lett. B252, 349 (1990)

13. Fendley, P., Lerche, W., Mathur, S. D., Warner, N. P.: Nucl. Phys. B348, 66 (1991)

14. Goddard, P., Nahm, W., Olive, D.: Phys. Lett. B160, 111 (1985)

15. Wolf, J. A.: J. Math. Mech. 14, 1033 (1965); Salamon, S.: Invent. Math. 67, 143 (1982)

Communicated by N. Yu. Reshetikhin 